\title{
有噪条件下的新类检测算法
}

\author{
曾凡霞 ${ }^{1,2)}$, 何泽文 ${ }^{1,2)}$, 张文生 ${ }^{1,2}$ * $^{*}$ \\ 1) (中国科学院自动化研究所复杂系统管理与控制国家重点实验室 北京 100190) \\ 2) (中国科学院大学 北京 100049) \\ (zhangwenshengia@hotmail.com)
}

\begin{abstract}
摘 要: 针对有噪条件下新类检测性能较差的问题, 提出一种基于核零空间判别局部保持投影算法(KNDLPP). 首先 通过核函数将样本隐式映射到高维特征空间，在核空间中利用距离加权机制对样本进行权重赋值，在保持局部结构 的同时降低离群噪声样本的影响; 然后利用样本类内零空间使同类样本啁塌为一点, 实现对已知类分布的有效约简; 最后基于零空间再求得使类间距最大化的变换矩阵, 以上步骤得到一个判别性变换矩阵以刻画样本的分布信息、描 述样本之间的相似性. 该算法能刻画样本潜在结构, 提升已知类与新未知类之间的判别性. 在 11 个公开数据集上的 实验结果表明, 该算法是有效和鲁棒的, 具有较好的新类检测性能. 在局部保持性实验中, KNDLPP 在 4 个 UCI 数据 集上的整体平均 AUC 值为 90.656\%; 在复杂结构保持性实验中, KNDLPP 在 Banana, Moon 及 3 个 UCI 数据集上的整 体平均 AUC 值为 $91.949 \%$; 在 2 个无噪高维数据集的新类检测实验中, KNDLPP 平均 AUC 值为 $86.214 \%$, 高于次优 算法 4 个百分点; 在 4 个 UCI 数据集的 4 种有噪条件下, KNDLPP 性能排名第 1 .
\end{abstract}

关键词：核方法；零空间；判别局部保持映射；新类检测

中图法分类号: TP391.41ＤOI: 10.3724/SP.J.1089.2021.18540

\section{A Novelty Detection Algorithm in the Presence of Noise}

\author{
Zeng Fanxia ${ }^{1,2)}$, He Zewen ${ }^{1,2)}$, and Zhang Wensheng ${ }^{1,2)^{*}}$ \\ 1) (The State Key Laboratory for Management and Control of Complex Systems, Institute of Automation, Chinese Academy of Sciences, Beijing 100190) \\ 2) (University of Chinese Academy of Sciences, Beijing 100049)
}

\begin{abstract}
To address the poor performance of novelty detection in the presence of noisy samples, a method named kernel null space discriminant locality preserving projections (KNDLPP) is proposed. Firstly, the training samples are transformed into a high dimensional space through a kernel function implicitly, and different weights are assigned to these samples according to the distance weighted scheme in the kernel space, to preserve the locality while reducing the impacts of noisy samples. Then, through the kernel null space of intra-class, each class collapses to a point, which makes each known class concise efficiently. Finally, a projection matrix maximizing the distance among inter-classes can be computed based on the null space, thus after these steps a discriminative transformation matrix is got to characterize the distribution and similarity of samples. This method can grasp the underlying structure of samples, and improve the discrimination between the known classes and the unknown novelty. The comparison experiments are based on eleven public datasets, the results validate the effectiveness and robustness of proposal during the testing, and this method performs well for novelty detection. During the experiments about locality preserving on 4 UCI
\end{abstract}

收稿日期: 2020-08-04; 修回日期: 2020-12-12. 基金项目：国家自然科学基金青年科学基金(61906190); 广东省重点领域研发计 划(2019B010153002). 曾凡霞(1988一), 女, 博士, 主要研究方向为机器学习、度量学习、重采样; 何泽文(1992一), 男, 博士研究生, 主要研究方向为深度学习、机器学习、目标检测与跟踪; 张文生(1965一), 男, 博士, 研究员, 博士生导师, CCF 会员, 论文通讯作者, 主要研究方向为人工智能、机器学习、大数据知识挖掘. 
UCI datasets, the whole mean AUC of KNDLPP is 90.656\%. During the experiments about complex structure on Banana, Moon and 3 UCI datasets, the whole mean AUC of KNDLPP is 91.949\%. During the experiments on 2 clean high dimensional datasets for novelty detection, the whole mean AUC of KNDLPP is 86.214\%, which is 4 percent higher than the second best algorithm. On 4 UCI datasets with 4 different kinds of noise, the performance of KNDLPP ranks first.

Key words: kernel methods; null space; discriminant local preserving projections; novelty detection

在给定只含已知类的训练样本下, 新类检测 ${ }^{[1]}$ 的目的在于从测试样本中检测出训练集中未出现 的未知类样本. 该类方法主要通过训练集中的已 知类样本建立一个学习器, 在测试过程中通过学 习器判断样本是否属于新的未知类别. 而学习器 对新未知类的有效检测程度可反映其在测试中的 鲁棒性程度. 随着社会各领域的多媒体化和数字 化发展，新类检测可实时地跟进应用动态、发现新 生事件，利于采取及时应对措施. 因此，新类检测 方法研究具有重要的研究价值与意义. 已有相关

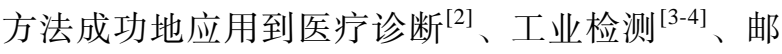

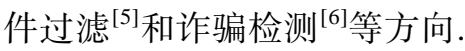

现有新类检测方法可分为统计方法和非统计 方法 2 类 $^{[7]}$. 统计方法根据已知类样本建立一个统 计准则, 通过该准则推断测试样本属于已知类分 布的概率. 该类方法基于某些统计假设前提展开, 但有 2 个方面原因使其不太适合复杂场景下高维 数据处理. 一方面, 实际应用数据成因复杂、维度 剧增, 真实情况可能致使维度灾难带来空空间现 象(empty space phenomenon ${ }^{[8]}$, 以致统计假设失 效; 另一方面, 统计方法可能涉及较多、较复杂的 参数变量, 使方法的灵活性较差. 而非统计类方法 在描述数据潜在分布方面相对更灵活.一个较好 的新类检测方法应该能够准确刻画已知类分布， 才可能实现对已知类和未知类之间的区分. 该类 方法常根据训练集学习样本的潜在结构信息, 以 描述已知类和未知类样本间的相似性.

在机器学习中, 可描述样本分布的学习方法 常分为全局信息保持和局部信息保持 2 类 $^{[9-10]}$. 基 于全局信息保持的方法以保持样本的全局结构为 目标来训练学习器, 以刻画样本之间的相似性, 将 测试集中与已知类不相似的样本判断为新的未知类. 基于 Foley-Sammon 变换(Foley-Sammon transform,

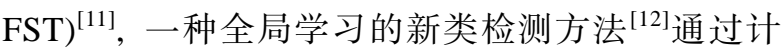
算类内样本的核零空间，使投影后的同类样本坍 塌为同一点，实现对已知类分布的有效约简. 但实 际数据结构复杂, 常存在一些特殊的局部结构或
离群噪声样本, 使全局类方法性能受到一定的负 面影响. 相对而言, 局部信息保持类方法在细节刻 画方面更加有效 ${ }^{[10]}$, 也可降低离群噪声样本的影 响. 尤其在多分类或有噪条件下，新类检测中的局 部结构相对全局结构更加重要 ${ }^{[13]}$. 若忽略局部结 构, 可能无法准确地刻画已知类的分布, 难以在测 试集中有效地识别新类样本.

针对上述缺点, 本文提出基于核零空间判别 局部保持投影(kernel null space discriminant locality preserving projections, KNDLPP)算法. 该算法 引人距离加权机制, 可有效地保持样本的局部结 构, 并控制噪声样本在学习过程中的影响, 提升学 习器对样本分布的刻画能力. 算法通过核函数将 已知类样本隐式映射到高维空间, 并利用核矩阵 计算类中心核矩阵和连接权重，克服了核空间的 表达和计算困难; 在核类内零空间下求得类间距 最大化的投影变换，集成最终的判别性变换矩阵. 该算法可对已知类别进行约简, 对类别具有较好 的判别性, 更易在测试过程中实现对新未知类的 检测. 基于 11 个公开数据集的 4 组实验从不同角 度进行了相关方法对比, 结果均验证了所提算法 在测试中的有效性和的鲁棒性, 具有较好的新类 检测性能.

\section{1 相关工作}

记 $\boldsymbol{X}=\left[\boldsymbol{x}_{1}, \boldsymbol{x}_{2}, \cdots, \boldsymbol{x}_{N}\right] \in \mathbb{R}^{d \times N}$ 为包含 $N$ 个具有 $d$ 维特征的训练样本集, $\boldsymbol{x}_{i}$ 表示第 $i$ 个训练样本. 该训练样本集所对应的类别为 $\boldsymbol{y}=\left[y_{1}, y_{2}, \cdots, y_{N}\right]$, $y_{i} \in\{1,2, \cdots, c\}$. 其中, $y_{i}$ 为 $\boldsymbol{x}_{i}$ 对应的类别标签, 属于已知 $c$ 个类别中的一类. 记待学习的特征空间 变换矩阵为 $\boldsymbol{P} \in \mathbb{R}^{d \times m}, m$ 为变换后样本的维度.

由于核方法是线性方法的拓展，下面首先简 单回顾与 KNDLPP 最相关的零 Foley-Sammon 变换 (null Foley-Sammon transform, NFST)和判别局部 保持投影(discriminant locality preserving projec- 
tions, DLPP) 2 种线性算法.

\subsection{NFST}

在 FST 的基础上, $\mathrm{NFST}^{[14]}$ 利用类内零空间学 习变换矩阵. 其中, $\mathrm{FST}^{[11]}$ 主要以最大化 Fisher 判别 准则(Fisher discriminant criterion)为学习目标, 即

$$
J\left(\boldsymbol{p}_{i}\right)=\frac{\boldsymbol{p}_{i}^{\mathrm{T}} \boldsymbol{S}_{\mathrm{b}} \boldsymbol{p}_{i}}{\boldsymbol{p}_{i}^{\mathrm{T}} \boldsymbol{S}_{\mathrm{w}} \boldsymbol{p}_{i}}
$$

最大的前提下计算正交变换矩阵 $\boldsymbol{P}=\left(\boldsymbol{p}_{1}, \boldsymbol{p}_{2}, \cdots, \boldsymbol{p}_{m}\right)$. 其中, $S_{\mathrm{b}}$ 为类间散度矩阵; $S_{\mathrm{w}}$ 为类内散度矩阵. 变换矩阵 $\boldsymbol{P}$ 的求解, 可通过关于矩阵 $\boldsymbol{S}_{\mathrm{b}}$ 和 $\boldsymbol{S}_{\mathrm{w}}$ 的 广义特征向量分解实现, 线性变换 $\boldsymbol{f}_{i}=\boldsymbol{P}^{\mathrm{T}} \boldsymbol{x}_{i}$ 则称 为 FST.

考虑高维小样本集或类内样本相似性较强可 能导致类内散度矩阵 $\boldsymbol{S}_{\mathrm{w}}$ 奇异的情况, NFST 将最 大化目标准则 $J\left(\boldsymbol{p}_{i}\right)$ 转为求解满足目标

$$
\left\{\begin{array}{l}
\boldsymbol{p}_{i}^{\mathrm{T}} \boldsymbol{S}_{\mathrm{w}} \boldsymbol{p}_{i}=0 \\
\boldsymbol{p}_{i}^{\mathrm{T}} \boldsymbol{S}_{\mathrm{b}} \boldsymbol{p}_{i}>0
\end{array}\right.
$$

的正交变换矩阵 $\boldsymbol{P}=\left(\boldsymbol{p}_{1}, \boldsymbol{p}_{2}, \cdots, \boldsymbol{p}_{m}\right)$. 其中, 等式说 明了矩阵 $\boldsymbol{P}$ 的向量 $\boldsymbol{p}_{i}$ 属于类内散度矩阵 $\boldsymbol{S}_{\mathrm{w}}$ 的零 空间 $\boldsymbol{Z}_{\mathrm{w}}$; 不等式说明了矩阵 $\boldsymbol{P}$ 的向量 $\boldsymbol{p}_{i}$ 属于类 间散度矩阵 $\boldsymbol{S}_{\mathrm{b}}$ 的非零空间. 由此, 2 个式子联立后 可使 Fisher 目标准则 $J\left(\boldsymbol{p}_{i}\right)=\infty$, 提升了其判别性.

假设训练样本满足类别平衡的条件, 用 $\boldsymbol{S}_{\mathrm{t}}$ 表 示总体散度矩阵, 则 3 个对称矩阵 $\boldsymbol{S}_{\mathrm{t}}, \boldsymbol{S}_{\mathrm{b}}$ 和 $\boldsymbol{S}_{\mathrm{w}}$ 之 间满足关系式 $\boldsymbol{S}_{\mathrm{t}}=\boldsymbol{S}_{\mathrm{w}}+\boldsymbol{S}_{\mathrm{b}}{ }^{[12]}, \boldsymbol{Z}_{\mathrm{t}} \subset \boldsymbol{Z}_{\mathrm{w}}$; 其中, $\boldsymbol{Z}_{\mathrm{t}}$ 为总体样本零空间. 对于满足式(2)中第 1 个条 件的投影向量 $\boldsymbol{p}_{i}$, 可推出 $\boldsymbol{p}_{i} \in\left(\boldsymbol{Z}_{\mathrm{t}}^{\perp} \cap \boldsymbol{Z}_{\mathrm{w}}\right)$; 其中, $\boldsymbol{Z}_{\mathrm{t}}^{\perp}$ 为总体样本零空间 $\boldsymbol{Z}_{\mathrm{t}}$ 的正交补空间. 因此, 变 换矩阵 $\boldsymbol{P}$ 的向量 $\boldsymbol{p}_{i}$ 可表示为 $\boldsymbol{Z}_{\mathrm{t}}^{\perp}$ 空间中基向量 $Q=\left\{\theta_{1}, \cdots, \theta_{n}\right\}$ 的线性组合 $p_{i}=A_{i 1} \boldsymbol{\theta}_{1}+\cdots+\boldsymbol{A}_{i n} \boldsymbol{\theta}_{n}$. 其 中, $\boldsymbol{A}_{i j}$ 为第 $i$ 个投影向量 $\boldsymbol{p}_{i}$ 关于基向量 $\boldsymbol{\theta}_{j}$ 的系数. 为了实现快速计算, 可先采用施密特正交化或标 准主成分变换计算 $\boldsymbol{Z}_{\mathrm{t}}^{\perp}$ 空间的基向量 $\boldsymbol{Q}=\left\{\boldsymbol{\theta}_{1}, \cdots, \boldsymbol{\theta}_{n}\right\}$; 然后转为求解满足 $\boldsymbol{A}^{\mathrm{T}} \boldsymbol{Q}^{\mathrm{T}} \boldsymbol{S}_{\mathrm{w}} \boldsymbol{Q} \boldsymbol{A}=\mathbf{0}$ 的系数矩阵 $\boldsymbol{A}$, 最后得到 NFST 的变换矩阵为 $\boldsymbol{P}=\boldsymbol{Q} \boldsymbol{A}$.

基于 NFST, 核零 Foley-Sammon 变换(kernel null Foley-Sammon transform, KNFST) ${ }^{[12]}$ 通过核函 数将样本隐式映射到更高维空间, 利用核类内零 空间学习已知类的潜在结构, 提升了其判别性. 该 工作尤其在单峰分布数据集中表现了较好的新类 检测性能. 文献[15-16]将其成功地应用于行人再
辨识 ${ }^{[17]}$ ，文献[18]利用零空间信息实现了对人脸的 识别, 这些应用现象也进一步表明了类内零空间 具有良好判别性. 然而, 当复杂数据集中存在离群 噪声样本或多峰分布时, 若对样本采用均匀权重, 相应性能会受到一定的影响.

\subsection{DLPP}

从样本关系的角度分析, KNFST 是一种全局 信息保持的方法. 但在新类检测中，训练集的局部 结构 ${ }^{[13]}$ 更重要. 学习器是否准确地保持样本的局 部结构, 决定了其能否准确地描述测试样本与已 知类样本的相似性, 影响着新类检测的性能. 因 此, 对新类检测而言, 局部结构信息是一个不容忽 略的因素. 在监督环境下, $\mathrm{DLPP}^{[19]}$ 利用标签信息 提升了非监督的局部保持投影(locality preserving projections, LPP) ${ }^{[20]}$ 的判别性. 区别于 FST, DLPP 利用距离加权机制对样本权重进行赋值, 更侧重 于保持数据的局部结构. 该工作将同时最小化类 内距离和最大化类间距离作为学习目标, 以得到 最优变换矩阵

$$
\boldsymbol{P}^{*}=\arg \min _{\boldsymbol{P}, \boldsymbol{P}^{\mathrm{T}} \boldsymbol{P}=\boldsymbol{I}} \frac{\operatorname{tr}\left(\boldsymbol{P}^{\mathrm{T}} \boldsymbol{X} \boldsymbol{L}_{\mathrm{w}} \boldsymbol{X}^{\mathrm{T}} \boldsymbol{P}\right)}{\operatorname{tr}\left(\boldsymbol{P}^{\mathrm{T}} \overline{\boldsymbol{X}} \boldsymbol{L}_{\mathrm{b}} \overline{\boldsymbol{X}}^{\mathrm{T}} \boldsymbol{P}\right)}
$$

其中, $\overline{\boldsymbol{X}}=\left[\overline{\boldsymbol{x}_{1}}, \overline{\boldsymbol{x}_{2}}, \cdots, \overline{\boldsymbol{x}_{c}}\right] \in \mathbb{R}^{d \times c}$ 为由 $c$ 个类中心向 量构成的矩阵; $\boldsymbol{L}_{\mathrm{b}}$ 和 $\boldsymbol{L}_{\mathrm{w}}$ 分别为对应本质图 (intrinsic graph) $\boldsymbol{G}_{\mathrm{w}}$ 和惩罚图 (penalty graph) $\boldsymbol{G}_{\mathrm{b}}$ 的 拉普拉斯矩阵. 本质图 $\boldsymbol{G}_{\mathrm{w}}=\left\{\boldsymbol{X}, \boldsymbol{W}_{\mathrm{w}}\right\}$ 和惩罚图 $\boldsymbol{G}_{\mathrm{b}}=\left\{\boldsymbol{X}, \boldsymbol{W}_{\mathrm{b}}\right\}$ 的连接矩阵 $\boldsymbol{W}_{\mathrm{w}}$ 和 $\boldsymbol{W}_{\mathrm{b}}$ 定义分别为

$$
\left\{\begin{array}{l}
\boldsymbol{W}_{\mathrm{w}}(i, j)= \begin{cases}\exp \left(\frac{-\left\|\boldsymbol{x}_{i}-\boldsymbol{x}_{j}\right\|^{2}}{t}\right), & \text { if } y_{i}=y_{j} \\
0, & \text { otherwise }\end{cases} \\
\boldsymbol{W}_{\mathrm{b}}(k, l)=\exp \left(\frac{-\left\|\overline{\boldsymbol{x}_{k}}-\overline{\boldsymbol{x}_{l}}\right\|^{2}}{t}\right)
\end{array}\right.
$$

其中, $\boldsymbol{L}_{\mathrm{w}}=\boldsymbol{D}_{\mathrm{w}}-\boldsymbol{W}_{\mathrm{w}}, \boldsymbol{D}_{\mathrm{w}}$ 为第 $i$ 个对角元素为 $\sum_{j=1}^{N} \boldsymbol{W}_{\mathrm{w}}(i, j)$ 的对角矩阵; $\boldsymbol{L}_{\mathrm{b}}=\boldsymbol{D}_{\mathrm{b}}-\boldsymbol{W}_{\mathrm{b}}, \boldsymbol{D}_{\mathrm{b}}$ 为第 $i$ 个对 角元素为 $\sum_{j=1}^{N} \boldsymbol{W}_{\mathrm{b}}(i, j)$ 的对角矩阵; $t$ 为由经验确定 的参数. 该模型仍可通过关于矩阵 $\boldsymbol{X} \boldsymbol{L}_{\mathrm{w}} \boldsymbol{X}^{\mathrm{T}}$ 和 $\bar{X} \boldsymbol{L}_{\mathrm{b}} \overline{\boldsymbol{X}}^{\mathrm{T}}$ 的广义特征向量分解求得变换矩阵 $\boldsymbol{P}$.

从目标函数分析, 无论对类内样本或类间样 本，DLPP 均采用距离加权机制，其本质在于同时 关注类内和类间的局部结构. 具体地, 同一类别 中, 距离越近的样本权重更高, 可保持同类样本的 
近邻关系; 而若不同类别的中心距离越近, 赋予更 高的权重可加大对近邻类别的惩罚程度, 可提升 类别的可分性. 因此, 通过这 2 点, 目标函数可提 升模型的判别性.

\section{2 本文算法}

新类检测通过对已知类样本的学习实现对未

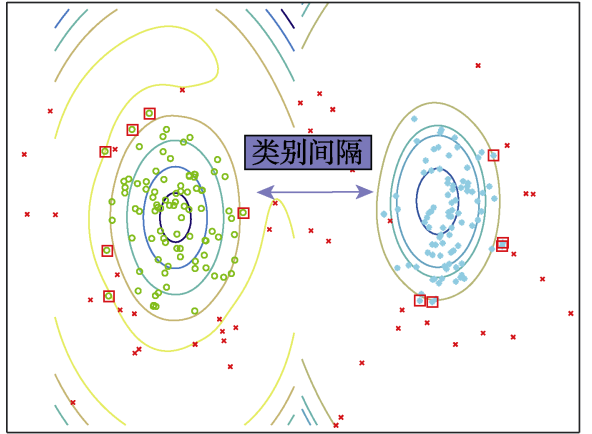

a. 真实分布
知类的检测, 其关键在于学习器能否准确地刻画 已知类分布. 然而, 由于实际数据结构复杂、特征 记录存在偏差等因素, 训练集中常存在一些离群 噪声样本影响学习器性能. 这一现象对全局类方 法的影响尤为严重. 如图 1 所示, 2 个已知类均含 有不同离群噪声样本. 若采用 NFST 的均匀权重机 制, 受噪声干扰, 图 1 中的所学分布与真实分布偏 差较大, 未知类样本可能被错分为已知类.

-已知类 -1 ; *已知类-2; 口 噪声样本; $\times$ 未知类

图 1 离群噪声样本对学习器的干扰分析图

\subsection{KNDLPP}

基于上述噪声的负面影响分析, 并结合 NFST 和 DLPP 的缺点分析可得知, 一个良好的新类检测方 法应该具备 3 个方面的特点: (1) 可有效地控制离群 样本的影响, 降低其对学习器的干扰程度; (2) 可刻 画数据复杂结构, 具有一定的非线性表达能力; (3) 具有较好的判别性，以提升新未知类与已知类之间 的可分性. 综合以上分析, 本文提出 KNDLPP 算法, 首先通过核函数 $\boldsymbol{K}\left(\boldsymbol{x}_{i}, \boldsymbol{x}_{j}\right)=\left\langle\Phi\left(\boldsymbol{x}_{i}\right), \Phi\left(\boldsymbol{x}_{j}\right)\right\rangle$ 将样本 $\boldsymbol{x}_{i}$ 和 $\boldsymbol{x}_{j}$ 隐式映射到高维空间, 可刻画数据非线性 的复杂结构; 然后采用与 DLPP 相似的距离加权机 制, 可有效地降低离群噪声样本的影响程度; 再利 用核类内零空间对已知类分布进行约简，可提升 对类别间的判别性. 因核空间变量可由该空间的 训练样本进行表达，其求解变换矩阵 $\boldsymbol{P}$ (在核空间 中的变换矩阵 $\boldsymbol{P}$ 大小应为 $N \times m)$ 的目标函数为

$$
\left\{\begin{array}{l}
\operatorname{tr}\left(\boldsymbol{P}^{\mathrm{T}} \boldsymbol{K} \boldsymbol{L}_{\mathrm{w}} \boldsymbol{K} \boldsymbol{P}\right)=0 \\
\max \operatorname{tr}\left(\boldsymbol{P}^{\mathrm{T}} \boldsymbol{K}_{\mathrm{U}} \boldsymbol{L}_{\mathrm{b}} \boldsymbol{K}_{\mathrm{U}}^{\mathrm{T}} \boldsymbol{P}\right)
\end{array}\right.
$$

其中, $\boldsymbol{K}$ 为训练样本的核矩阵; $\boldsymbol{K}_{\mathrm{U}}$ 为关于训练样 本与类中心的核矩阵; $\boldsymbol{L}_{\mathrm{w}}$ 和 $\boldsymbol{L}_{\mathrm{b}}$ 分别为核空间中 的本质图 $\boldsymbol{G}_{\mathrm{w}}=\left\{\boldsymbol{X}, \boldsymbol{W}_{\mathrm{w}}\right\}$ 和惩罚图 $\boldsymbol{G}_{\mathrm{b}}=\left\{\boldsymbol{X}, \boldsymbol{W}_{\mathrm{b}}\right\}$ 的拉 普拉斯矩阵, 连接矩阵 $\boldsymbol{W}_{\mathrm{w}}$ 和 $\boldsymbol{W}_{\mathrm{b}}$ 的定义为

$$
\begin{cases}\boldsymbol{W}_{\mathrm{w}}(i, j)= \begin{cases}\mathrm{e}^{\left(\frac{-\left\|\Phi\left(x_{i}\right)-\Phi\left(x_{j}\right)\right\|^{2}}{t}\right)}, & \text { if } y_{i}=y_{j} \\ 0, & \text { otherwise }\end{cases} \\ \boldsymbol{W}_{\mathrm{b}}(k, l)=\mathrm{e}^{\left(\frac{-\left\|\overline{\boldsymbol{\Phi}_{k}}-\overline{\boldsymbol{\Phi}_{\boldsymbol{T}}}\right\|^{2}}{t}\right)}\end{cases}
$$

其中, $\overline{\Phi_{k}}$ 和 $\overline{\boldsymbol{\Phi}}$ 分别表示核空间中第 $k$ 类和第 $j$ 类 的均值中心向量. 由于从原空间到高维空间的映 射函数 $\Phi(x)$ 是隐式的, 该模型无法像 DLPP 一样 直接求解, 下面将针对该模型进行求解.

\section{2 模型求解}

不同于 KNFST 和 DLPP 这 2 种算法，当核函 数 $\Phi(\boldsymbol{x})$ 将样本隐式映射到高维空间时, KNDLPP 的计算和求解面临 2 个难点: (1) 对于 $\boldsymbol{K}_{\mathrm{U}}$, 核函数 的隐式映射使核空间的类均值中心无法直接显式 计算; (2)对于权重 $\boldsymbol{W}_{\mathrm{w}}$ 和 $\boldsymbol{W}_{\mathrm{b}}$, 本质图和惩罚图的 连接权重需根据核空间中的距离进行计算. 为了 解决这 2 个问题, 下面先对相关计算进行推导.

先针对第 1 点, 展开对类中心计算的讨论. 由 于核空间中任意向量均可由训练样本向量的线性 组合进行表达，记 $n_{l}$ 为第 $l$ 类的样本数，则核空间 中第 $l$ 类的均值中心向量为 $\overline{\boldsymbol{\Phi}_{1}}=\frac{1}{n_{l}} \sum_{y_{j}=l} \Phi\left(\boldsymbol{x}_{j}\right)$. 根 据训练集 $\left[x_{1}, x_{2}, \cdots, x_{N}\right]$ 所对应的核矩阵 
$\boldsymbol{K}\left(\boldsymbol{x}_{i}, \boldsymbol{x}_{j}\right)=\left\langle\Phi\left(\boldsymbol{x}_{i}\right), \Phi\left(\boldsymbol{x}_{j}\right)\right\rangle$, 核空间里各类均值中 心 $\overline{x_{l}}$ 与训练样本 $x_{i}$ 的内积矩阵可表达为

$$
\boldsymbol{K}_{\mathrm{U}}\left(\boldsymbol{x}_{i}, \overline{\boldsymbol{x}_{l}}\right)=\left\langle\Phi\left(\boldsymbol{x}_{i}\right), \overline{\boldsymbol{\Phi}_{l}}\right\rangle=\left\langle\Phi\left(\boldsymbol{x}_{i}\right), \frac{1}{n_{l}} \sum_{y_{j}=l} \Phi\left(\boldsymbol{x}_{j}\right)\right\rangle .
$$

因此，可计算矩阵 $\boldsymbol{K}_{\mathrm{U}}$ 为

$$
\boldsymbol{K}_{\mathrm{U}}\left(\boldsymbol{x}_{i}, \overline{\boldsymbol{x}_{l}}\right)=\frac{1}{n_{l}} \sum_{y_{j}=l} \boldsymbol{K}\left(\boldsymbol{x}_{i}, \boldsymbol{x}_{j}\right)
$$

通过样本核内积矩阵 $\boldsymbol{K}$, 实现了对矩阵 $\boldsymbol{K}_{\mathrm{U}}$ 的 计算, 由此解决了第 1 个难点.

对于第 2 点，先考虑类中心权重的计算，其关 键在于各中心间距离的计算. 将类中心距离公式 展开，并代人核矩阵 $\boldsymbol{K}$ 的元素得到

$$
\begin{aligned}
& \left\|\overline{\boldsymbol{\Phi}_{k}}-\overline{\boldsymbol{\Phi}}\right\|^{2}=\left\|\frac{1}{n_{k}} \sum_{y_{j}=k} \Phi\left(\boldsymbol{x}_{j}\right)-\frac{1}{n_{l}} \sum_{y_{j}=l} \Phi\left(\boldsymbol{x}_{j}\right)\right\|= \\
& \frac{1}{n_{k}^{2}} \sum_{y_{j_{1}}=k} \sum_{y_{j_{2}}=k} \Phi\left(\boldsymbol{x}_{j_{1}}\right) \Phi\left(\boldsymbol{x}_{j_{2}}\right)- \\
& \frac{2}{n_{k} n_{l}} \sum_{y_{j_{3}}=k} \sum_{y_{j_{4}}=l} \Phi\left(\boldsymbol{x}_{j_{3}}\right) \Phi\left(\boldsymbol{x}_{j_{4}}\right)+ \\
& \frac{1}{n_{l}^{2}} \sum_{y_{j_{5}}=l} \sum_{y_{j_{6}}=l} \Phi\left(\boldsymbol{x}_{j_{5}}\right) \Phi\left(\boldsymbol{x}_{j_{6}}\right)= \\
& \frac{1}{n_{k}^{2}} \sum_{y_{j_{1}}=k} \sum_{y_{j_{2}}=k} \boldsymbol{K}\left(\boldsymbol{x}_{j_{1}}, \boldsymbol{x}_{j_{2}}\right)- \\
& \frac{2}{n_{k} n_{l}} \sum_{y_{j_{3}}=k} \sum_{y_{j_{4}}=l} \boldsymbol{K}\left(\boldsymbol{x}_{j_{3}}, \boldsymbol{x}_{j_{4}}\right)+ \\
& \frac{1}{n_{l}^{2}} \sum_{y_{j_{5}}=l} \sum_{y_{j_{6}}=l} \boldsymbol{K}\left(\boldsymbol{x}_{j_{5}}, \boldsymbol{x}_{j_{6}}\right)
\end{aligned}
$$

与式(8)展开原理相似, 核空间的类内样本距 离为

$$
\begin{aligned}
& \left\|\Phi\left(x_{i}\right)-\Phi\left(x_{j}\right)\right\|^{2}= \\
& \Phi^{2}\left(x_{i}\right)-2 \Phi\left(x_{i}\right) \Phi\left(x_{j}\right)+\Phi^{2}\left(x_{j}\right)= \\
& \boldsymbol{K}\left(\boldsymbol{x}_{i}, \boldsymbol{x}_{i}\right)-2 \boldsymbol{K}\left(\boldsymbol{x}_{i}, \boldsymbol{x}_{j}\right)+\boldsymbol{K}\left(\boldsymbol{x}_{j}, \boldsymbol{x}_{j}\right)
\end{aligned}
$$

将式(8)(9)代人式(6), 可通过训练样本核矩阵 $\boldsymbol{K}$ 计算拉普拉斯矩阵 $\boldsymbol{L}_{\mathrm{b}}$ 和 $\boldsymbol{L}_{\mathrm{w}}$. 由此解决了第 2 个 难点.

得到以上计算后, 观察式(5), 应先求解类内 矩阵 $\boldsymbol{K} \boldsymbol{L}_{\mathrm{w}} \boldsymbol{K}$ 的零空间变换矩阵 $\boldsymbol{B}$, 进一步转为在 该零空间中求解满足目标的变换矩阵 $\boldsymbol{C}$, 即

$$
\max _{\boldsymbol{C}, \boldsymbol{C}^{\mathrm{T}} \boldsymbol{C}=\boldsymbol{I}} \operatorname{tr}\left(\boldsymbol{C}^{\mathrm{T}} \boldsymbol{B}^{\mathrm{T}} \boldsymbol{K}_{\mathrm{U}} \boldsymbol{L}_{\mathrm{b}} \boldsymbol{K}_{\mathrm{U}}^{\mathrm{T}} \boldsymbol{B} \boldsymbol{C}\right) .
$$

其中, 变换矩阵的求解可简化为特征向量分解问
题，最后集成得到变换矩阵 $\boldsymbol{P}=\boldsymbol{B C}$.

由此, 测试中可根据所得矩阵 $\boldsymbol{P}$ 计算新的测试 样本 $\boldsymbol{x}_{q}$ 与训练样本之间的距离相似性, 判断该样本 是属于已知类还是未知类. 具体地, 得到矩阵 $\boldsymbol{P}$ 后, 测试样本 $\boldsymbol{x}_{q}$ 在核空间中的投影为 $\boldsymbol{k}_{q}^{\mathrm{T}} \boldsymbol{P}$. 其中, $\boldsymbol{k}_{q}$ 为 测试样本 $\boldsymbol{x}_{q}$ 与 $N$ 个训练样本 $\boldsymbol{X}=\left[\boldsymbol{x}_{1}, \boldsymbol{x}_{2}, \cdots, \boldsymbol{x}_{N}\right]$ 的 核内积向量. 则在核空间中, 测试样本 $\boldsymbol{x}_{q}$ 与训练 样本 $\boldsymbol{x}_{i}$ 之间的距离为 $\left\|\boldsymbol{k}_{i}^{\mathrm{T}} \boldsymbol{P}-\boldsymbol{k}_{q}^{\mathrm{T}} \boldsymbol{P}\right\|$. 与 $\boldsymbol{k}_{q}$ 类似, $\boldsymbol{k}_{i}$ 为训练样本 $\boldsymbol{x}_{i}$ 与 $N$ 个训练样本 $\boldsymbol{X}=\left[\boldsymbol{x}_{1}, \boldsymbol{x}_{2}, \cdots, \boldsymbol{x}_{N}\right]$ 的核内积向量, 可由核矩阵 $\boldsymbol{K}$ 取行向量而得. 整 理以上求解过程, KNDLPP 算法步骤如下.

输人.训练样本 $X=\left[x_{1}, x_{2}, \cdots, x_{N}\right]$ 及其对应的 标签 $\boldsymbol{y}=\left[y_{1}, y_{2}, \cdots, y_{N}\right]$, 根据设定的核函数得到核 矩阵 $\boldsymbol{K}$.

输出. 变换矩阵 $\boldsymbol{P}=\boldsymbol{B C}$, 用以计算每个测试样 本 $\boldsymbol{x}_{q}$ 与训练样本 $\boldsymbol{x}_{i}$ 之间的距离 $\left\|\boldsymbol{k}_{i}^{\mathrm{T}} \boldsymbol{P}-\boldsymbol{k}_{q}^{\mathrm{T}} \boldsymbol{P}\right\|$, 根据 距离相似性检测出新的未知类样本.

Step1. 根据式(7)计算核类中心矩阵 $\boldsymbol{K}_{\mathrm{U}}$.

Step2. 根据训练样本核矩阵 $\boldsymbol{K}$, 分别将式(8)(9)代 人式(6), 计算基于本质图和惩罚图的拉普拉斯矩阵 $\boldsymbol{L}_{\mathrm{w}}$ 和 $\boldsymbol{L}_{\mathrm{b}}$.

Step3. 计算核类内矩阵 $\boldsymbol{K} \boldsymbol{L}_{\mathrm{w}} \boldsymbol{K}$ 的零空间变换矩阵 $\boldsymbol{B}$.

Step4. 对通过特征向量分解, 计算满足目标函数 $\max _{C, C^{\mathrm{T}} C=I} \operatorname{tr}\left(\boldsymbol{C}^{\mathrm{T}} \boldsymbol{B}^{\mathrm{T}} \boldsymbol{K}_{\mathrm{U}} \boldsymbol{L}_{\mathrm{b}} \boldsymbol{K}_{\mathrm{U}}^{\mathrm{T}} \boldsymbol{B} \boldsymbol{C}\right)$ 的变换矩阵 $\boldsymbol{C}$.

Step5. 计算最终变换矩阵 $\boldsymbol{P}=\boldsymbol{B C}$.

不同于 DLPP, KNDLPP 合理地利用了零空间 信息, 使同类样本尽可能坉塌为一点, 对已知类的 分布进行了约简, 从类内角度提升了新类检测的 判别性. 此外, KNDLPP 利用核函数的优势将样 本扩展到高维空间, 克服了实际数据较难满足线 性表达的缺点, 可刻画数据的复杂结构. 综合这 2 点, KNDLPP 比 DLPP 具有更好的判别性和表达 能力.

区别于全局类方法 KNFST, KNDLPP 不仅具 有较好的局部保持性，且最大化类间距可提升样 本坍塌后的类别可分性. 这种区别使 KNDLPP 比 KNFST 更适合于新类检测. 如图 2 所示, KNFST 采用均匀权重, 可能使呈多峰分布的已知类 2 坍塌 为一点. 对原空间距离已知类较远的未知类样本而 言, KNFST 可能使投影后的未知类与已知类距离较 近, 使部分新类样本被误判为已知类. 而 KNDLPP 


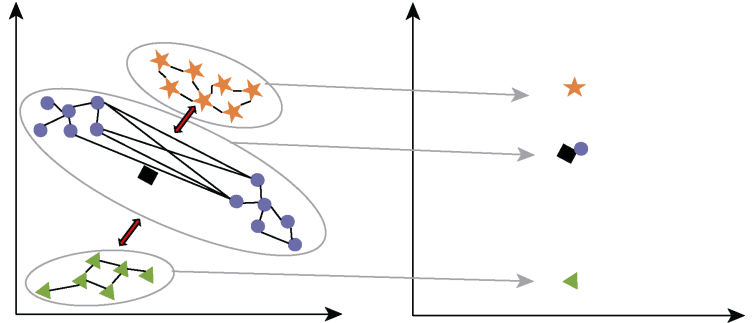

a. KNFST

$\star$ 已知类-1; 已知类- $2 ;$ 已知类-3;

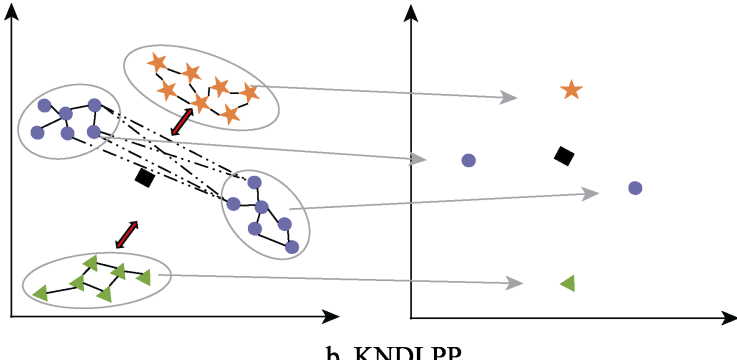

b. KNDLPP

$-\cdot$ 弱连接权

图 $2 \mathrm{KNFST}$ 与 KNDLPP 在新类检测中的对比

引人了距离加权机制，降低了已知类 2 中 2 个子簇 之间的连接. 与此同时, KNDLPP 最大化类间距, 尽可能地提升了类别间的可分性. 因此, 与 KNFST 相比, KNDLPP 算法能更准确地描述已知 类分布，有利于新类检测中提升已知类与未知类 之间的区分性.

\section{3 实验及结果分析}

为了验证所提 KNDLPP 在新类检测中的有效 性, 实验基于 7 个 UCI 数据集 ${ }^{[21]}$, 以及 Banana, Moon, Binalpha_uni 和 MSRA25_uni ${ }^{\mathbb{1}} 4$ 个数据集 进行了 4 组新类检测实验. 实验 1 和实验 2 分别从 2 种算法性质角度进行对比, 实验 3 和实验 4 分别 基于无噪条件和有噪条件进行对比.

实验中, 训练样本均为已知类, 测试样本同时 包含已知类和未知类. 实验对训练中的已知类样 本集按类别进行随机对半划分, 一半用作训练, 一 半与未知类一起用作测试. 诸如 KNDLPP 一类求 变换矩阵的方法, 均先在训练集中学习一个变换 矩阵, 后根据变换矩阵计算每个测试样本与所有 训练样本间的距离相似性, 根据最小距离值判断 其是否为新的未知类样本. 参考新类检测中评测 标准 ${ }^{[22]}$, 实验主要从 ROC 曲线和 AUC 值 2 个角度 进行性能评价.

\section{1 局部保持性实验}

KNDLPP 和 KNFST 除了权重机制不同以外, 也有着本质的差异. 不同于 KNFST, KNDLPP 采用 距离加权机制对样本进行连接权重赋值, 因而具 有局部保持性的优势. 为了验证 KNDLPP 的这一 优势, 实验选取了 Diabetes, Ionosphere, Iris 和 Vowel 这 4 个 UCI 数据集对 2 种算法进行对比. 数据集的 具体信息如表 1 所示.
表 1 实验 1 所使用的 4 个 UCI 数据集信息

\begin{tabular}{lcccc}
\hline \multicolumn{1}{c}{ 数据集 } & 样本数 & 特征数 & 类别数 & 新类别 ID \\
\hline Diabetes & 768 & 8 & 2 & 2 \\
Ionosphere & 351 & 34 & 2 & 2 \\
Iris & 150 & 4 & 3 & 1 \\
Vowel & 990 & 10 & 11 & $4,7,8,9$ \\
\hline
\end{tabular}

由于不同核函数适合不同数据集，为避免该 因素影响实验公正性, 该组实验的核函数均采用 线性核, 以便更直观地分析 2 种算法在局部保持性 方面的差异. 对已知类中存在多个类别的情况, 新 类检测常做 2 种处理: (1) 将多个已知类看做一类 进行单类学习; (2) 保持原有标签进行多类学习. 考虑这一情况, 在 Iris 和 Vowel 这 2 个含有多个已 知类的数据集上分别都进行了单类和多类的实验, 在另外 2 个数据集上仅做单类实验. 为了避免训练 集的随机划分影响实验公正性, 数据划分过程重 复 100 次, 实验取其平均结果进行报告. 最终的平 均 AUC 值如表 2 所示, 平均 ROC 曲线如图 3 所示.

分析表 2 的平均 AUC 值, 与 KNFST 相比, KNDLPP 的性能具有较明显的优势. 针对存在多 类的数据集, KNFST 的平均 AUC 值在 Iris 单类情 况下相对多类情况低了约 35\%. 这一现象的原因 在于, KNFST 仅依靠标签信息进行学习, 忽略了数

表 2 实验 1 的平均 AUC 值 $\%$

\begin{tabular}{lcc}
\hline \multicolumn{1}{c}{ 数据集 } & KNDLPP & KNFST \\
\hline Diabetes & 76.139 & 58.949 \\
Ionosphere & 95.759 & 81.921 \\
Iris-单类 & 98.254 & 59.091 \\
Iris-多类 & 99.744 & 94.807 \\
Vowel-单类 & 85.491 & 53.563 \\
Vowel-多类 & 88.549 & 50.236 \\
\hline 平均 & 90.656 & 66.428 \\
\hline
\end{tabular}




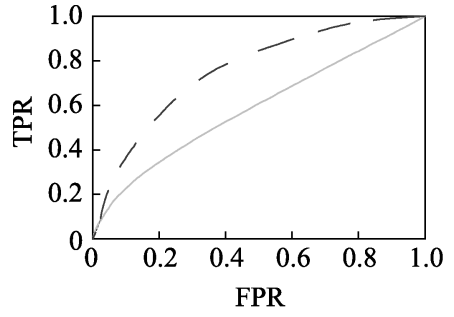

a. Diabetes

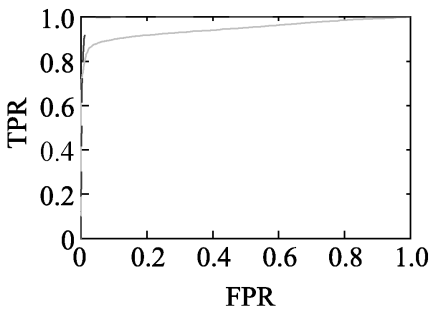

d. Iris-多类

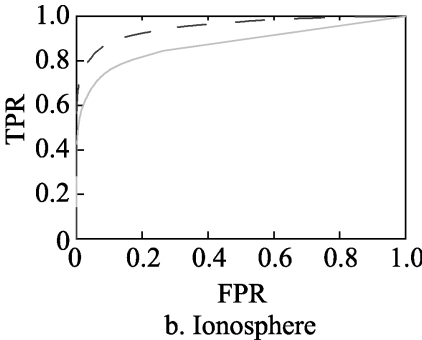

b. Ionosphere

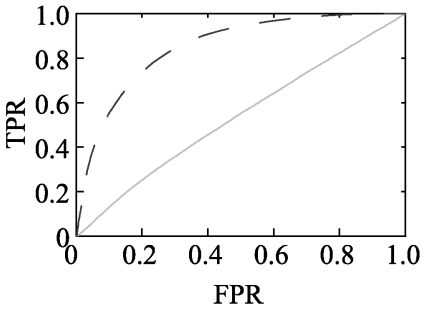

e. Vowel-单类
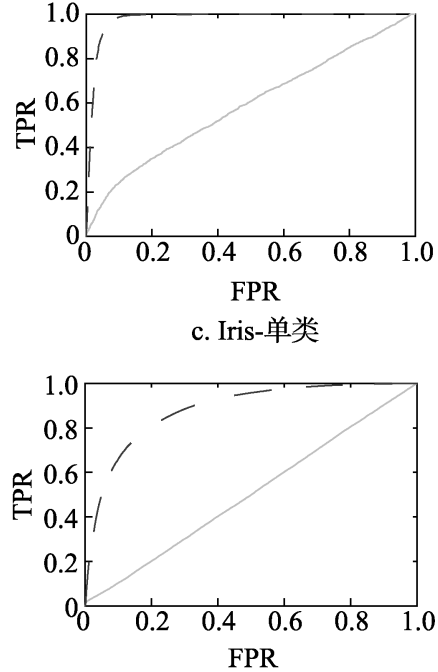

f. Vowel-多类

KNFST; - - KNDLPP

图 $3 \mathrm{KNDLPP}$ 与 KNFST 的 ROC 曲线对比

据的局部结构性. 而 KNDLPP 的平均 AUC 值在多 类情况下结果依然均优于单类情况，且 2 种情况的 差异较小. 该现象说明, KNDLPP 较好地刻画了 数据的局部结构, 使性能在 2 种情况下较为稳定.

从图 3 的 ROC 曲线可看出, KNDLPP 在 4 个 数据集上取得了全面优势. 对比分析 2 种算法, 其 差异主要体现在 3 个方面. 首先体现在类内样本连 接权重上：作为全局方法, KNFST 不论距离远近, 均对同类样本赋予相同权重; 而 KNDLPP 对同类 样本连接权重的赋值与其距离成反比, 距离越近 的样本，权重越大，以侧重于保持同类样本的局部 结构性. 其次体现在各类均值中心的连接权重上: KNFST 对所有不同的类中心均赋予相同权重; KNDLPP 的类中心连接权重仍与中心间的距离成 反比, 其目的在于对近邻的 2 个类别赋予更大的惩 罚程度，以提升变换后的类别可分性. 最后体现在 进行类内零空间变换后, KNDLPP 要求不同类的坡 塌点距离尽可能大，以进一步提升算法的判别性; 而 KNFST 则无该方面的要求. 这 3 个方面的区别 使 KNDLPP 能更好地学习已知类的局部结构, 具 有较强的判别性, 提升测新类检测性能.

\section{2 复杂结构保持性实验}

考虑 KNDLPP 与 DLPP 之间的联系, 实验 2 着重验证 KNDLPP 对数据的复杂结构保持性. 实 验基于 Banana, Statlog(heart), Moon, Seeds 和 Glass 这 5 个数据集对 2 种算法进行对比. 其中, Stat$\log$ (heart), Seeds 和 Glass 属于 UCI 数据集, Banana 和 Moon 来自工具包 ${ }^{[1]} 2$ 个非线性结构数据集. 数
据集的具体信息如表 3 所示.

表 3 实验 2 所使用的 5 个数据集信息

\begin{tabular}{lcccc}
\hline \multicolumn{1}{c}{ 数据集 } & 样本数 & 特征数 & 类别数 & 新类别 ID \\
\hline Banana & 400 & 2 & 2 & 1 \\
Statlog(heart) & 270 & 13 & 2 & 2 \\
Moon & 400 & 2 & 2 & 1 \\
Seeds & 210 & 7 & 3 & 3 \\
Glass & 214 & 9 & 6 & 5,6 \\
\hline
\end{tabular}

实验中 KNDLPP 的核函数统一选取高斯核. 针对含有多个已知类的 Seeds 和 Glass 数据集, 实 验依旧进行了单类和多类 2 种情况的对比. 训练集 的划分过程重复 100 次, 平均 AUC 值如表 4 所示, 平均 ROC 曲线如图 4 所示.

表 4 实验 2 的平均 AUC 值结果 $\%$

\begin{tabular}{lcc}
\hline 数据集 & KNDLPP & DLPP \\
\hline Banana & 99.219 & 92.864 \\
Statlog (heart) & 81.347 & 67.216 \\
Moon & 98.953 & 91.074 \\
Seeds-单类 & 93.264 & 89.323 \\
Seeds-多类 & 92.657 & 82.714 \\
Glass-单类 & 89.727 & 86.868 \\
Glass-多类 & 88.473 & 89.830 \\
\hline 平均 & 91.949 & 85.698 \\
\hline
\end{tabular}

观察表 4 的平均 AUC 值, KNDLPP 整体性能 优于 DLPP. 其中, 在 Seeds 数据集的多类情况下, KNDLPP 的平均 AUC 值比 DLPP 高出约 10\%; 在 Statlog(heart)数据集上, KNDLPP 的平均 AUC 值高 
出 DLPP 约 $14 \%$. 针对同一数据集的单类和多类 2 种情况, KNDLPP 的内部差异相对较小, 而在 Seeds 数据集上, DLPP 2 种情况下的平均 AUC 值 差异达到约 $7 \%$. 对于 Glass 数据集的多类情况下， DLPP 的平均 AUC 值比 KNDLPP 略高, 说明线性 结构可能更适合该数据集特性，也证实了不同数 据集有不同的数据结构.
由图 4 中的 ROC 曲线可知, KNDLPP 大部分 情况下性能均优于 DLPP. 众所周知, Banana 和 Moon 这 2 个数据集属于经典的非线性结构数据集, 能更好地测试算法对数据复杂结构的刻画能力. ROC 曲线结果表明，由于同时利用了类内零空间 与核技巧的优势，与 DLPP 相比, KDNLPP 对复杂 数据结构具有更好的适应性.
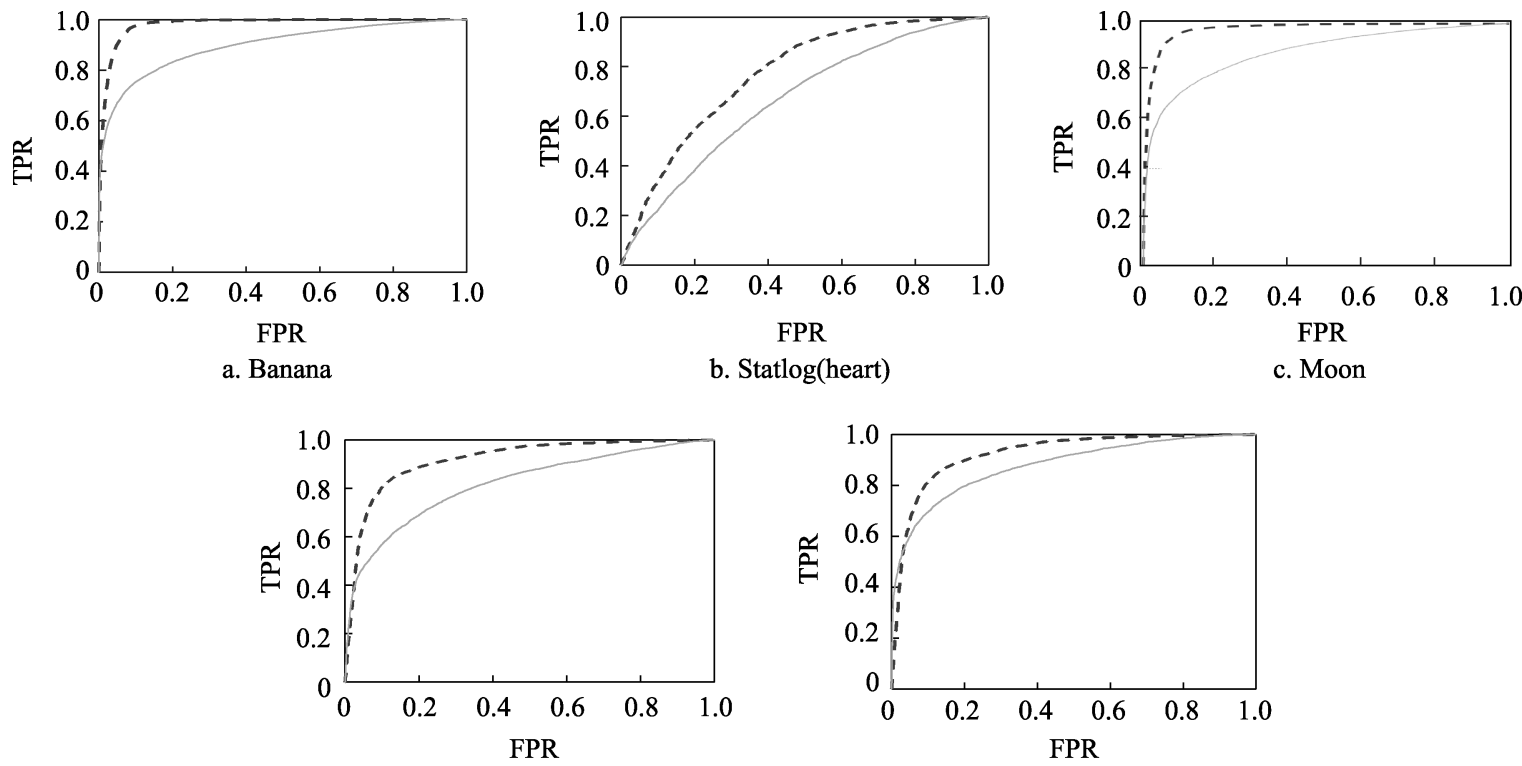

d. Seeds-单类
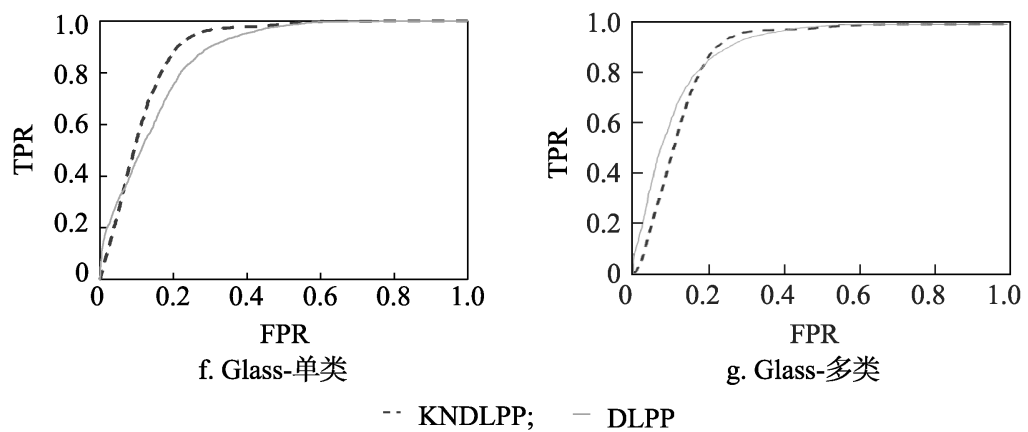

图 4 KNDLPP 与 DLPP 的 ROC 曲线对比

\section{3 无噪条件下的新类检测实验}

为全面验证 KNDLPP 的新类检测性能, 除了 KNFST 和 DLPP 以外, 实验 3 选取了核方法 $\mathrm{KSR}^{[23]}$ 、新类检测常用经典方法 $\mathrm{iForest}^{[24]}$, RKDE (robust kernel density estimator) ${ }^{[25]}$, LOF(local outlier factor ${ }^{[26]}$ 作为对比方法. 虽然 LOF, RKDE 和 iForest 属于非监督方法, 但在新类检测中均具有 较好的性能, 常与监督方法进行比较. 实验数据选 取了 Binalpha_uni 和 MSRA25_uni 这 2 个数据集, 具体数据信息如表 5 所示.

实验对每个数据集取前一半类别作已知类， 依旧在每个已知类中随机选取一半的样本用作训
表 5 实验 3 所使用数据集信息

\begin{tabular}{ccccc}
\hline 数据集 & 样本数 & 特征数 & 类别数 & 新类别 ID \\
\hline Binalpha_uni & 1404 & 320 & 36 & $19 \sim 36$ \\
MSRA25_uni & 1799 & 256 & 12 & $7 \sim 12$ \\
\hline
\end{tabular}

练, 其余已知类和未知类样本用作测试. 为避免随 机性, 数据划分过程重复 20 次, 取平均结果进行 报告. 本次实验对 2 个数据集均仅作单类讨论，即 将所有已知类别均作一类处理. 实验的平均 AUC 值如表 6 所示.

观察表 6 可知, KNDLPP 在同类方法中取得了 较为明显的优势. 而新类检测经典方法 iForest, 
RKDE 和 LOF 这三者性能较差. 该现象的原因在 于：其均在原欧几里得空间或所投影的核空间进 行数据分析时, 忽略了所在空间的特征关联性和 穴余性. 而 KSR 与 KNFST 这两者虽均采用均匀权 重, 但其中的差异在于 KNFST 采用了零空间信息. 分析 MSRA25_uni 数据集上的结果, KSR 性能甚至 优于 KNFST，其原因在于 KNFST 侧重于全局结构 下的类内零空间, 导致其表达能力有限. 对比 MSRA25_uni 数据集上的结果, 由于在局部保持性 和判别性 2 个方面的不同, KNFST 与 KNDLPP 的 差异达到了 15\%. 而 DLPP 的平均 AUC 值虽低于 KNDLPP，但得益于其具有的局部保持性，前者仍 然具有较好性能. 对比 KNFST 和 DLPP 这两者发 现，单依赖零空间或局部保持性，并不一定能够在 所有数据集上取得最优性能，这一现象侧面表明 了 KNDLPP 的优势综合来自零空间、局部保持性
和复杂结构保持性这 3 个方面. 以上分析表明，在 无噪条件下, KNDLPP 在测试中具有良好的新类检 测性能.

\section{4 有噪条件下的新类检测实验}

为了进一步全面验证 KNDLPP 能否有效控制 离群噪声样本, 提升新类检测性能, 本节在 Glass, Seeds, Ionosphere 和 Vowel 4 个 UCI 数据集上进 行加噪实验. 原始数据集信息与第 3.1 节和第 3.2 节一致. 已知类与未知类的生成方式与第 3.3 节一 致. 实验采用了 2 种加噪方式: 第 1 种方式为将数 据集的每个特征模标准化为 1 , 再分别随机将数据 矩阵中 $10 \%, 20 \%$ 和 $30 \%$ 的元素置为 0 或 1; 第 2 种 方式将扩充噪声作为特征维度, 扩展维度数为原始 特征维数的 $10 \%$. 对比方法与第 3.3 节一致, 整个实 验关于训练集的划分过程重复 20 次, 其平均 ROC 曲线如图 5 图 8 所示, 平均 AUC 值见各图右下角.

表 6 实验 3 的平均 AUC 值结果

\begin{tabular}{lcccccccc}
\hline \multicolumn{1}{c}{ 数据集 } & iForest & RKDE & LOF & KSR & KNFST & DLPP & KNDLPP \\
\hline Binalpha_uni & 68.018 & 62.213 & 71.874 & 66.996 & 67.260 & 65.273 \\
MSRA25_uni & 67.730 & 58.018 & 83.176 & 97.009 & 83.372 & 96.676 & 98.804 \\
\hline 平均 & 67.874 & 60.116 & 77.525 & 82.003 & 75.316 & 80.975 \\
\hline
\end{tabular}
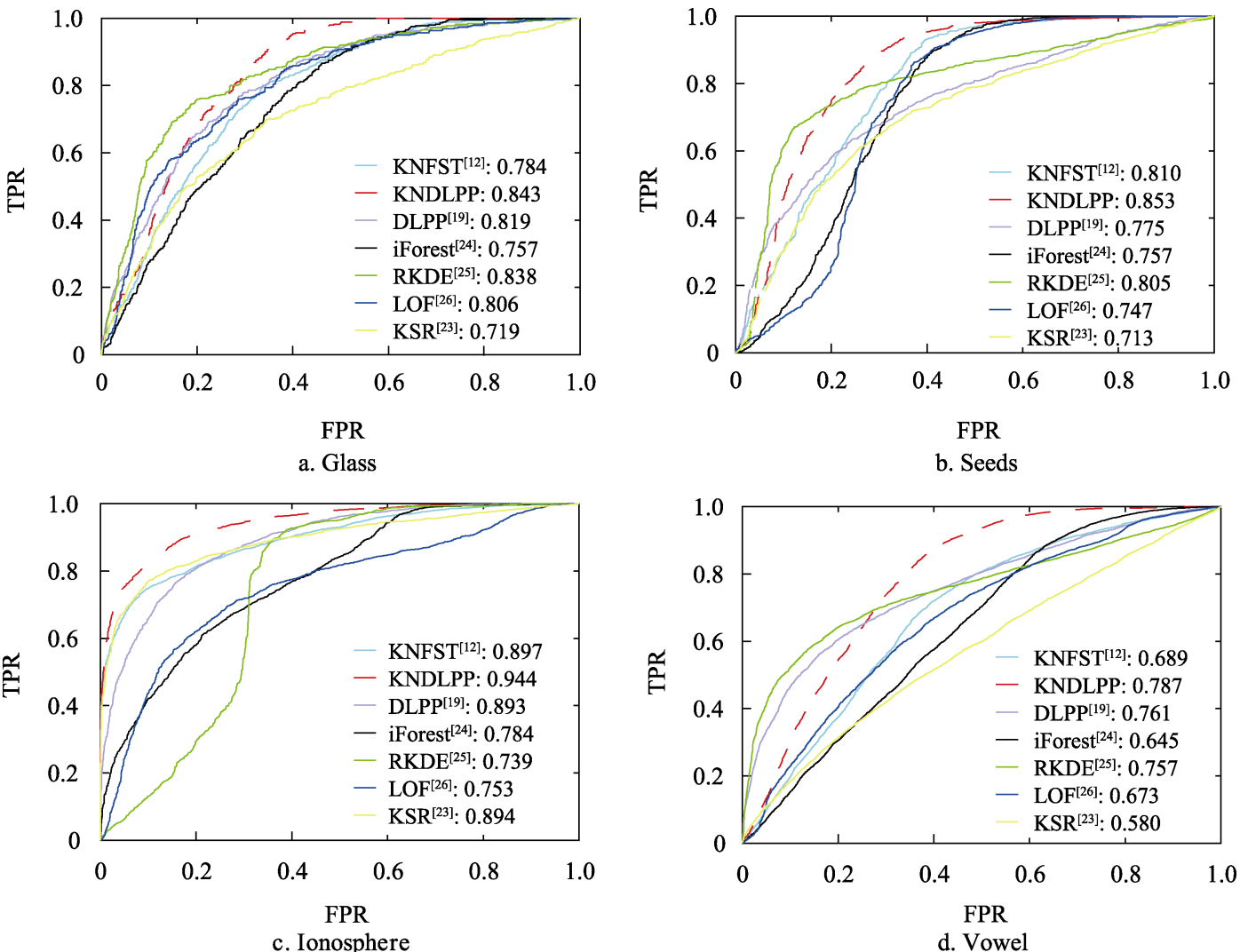

图 5 UCI 数据集 $10 \%$ 噪声程度下的 ROC 曲线及平均 AUC 值对比 

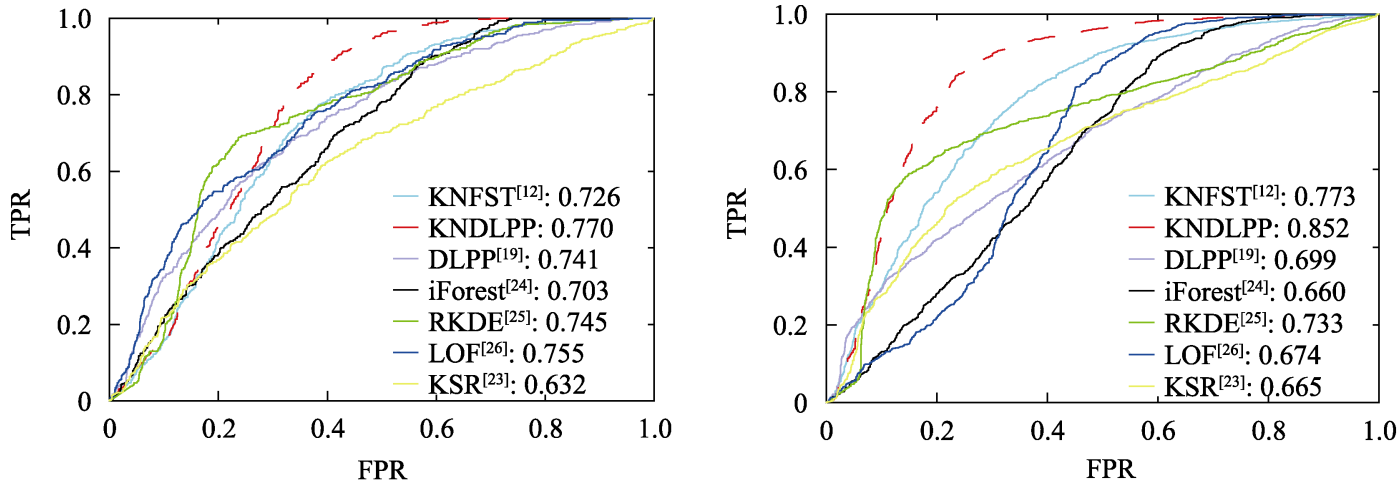

a. Glass

b. Seeds
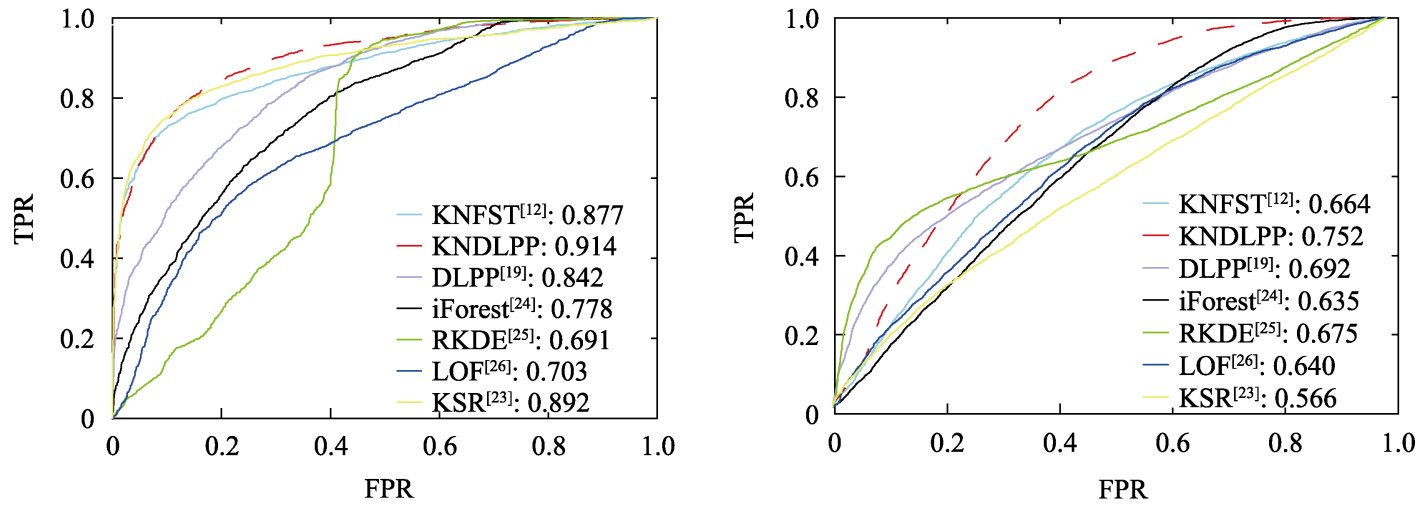

c. Ionosphere

d. Vowel

图 6 UCI 数据集 $20 \%$ 噪声程度下的 ROC 曲线及平均 AUC 值对比
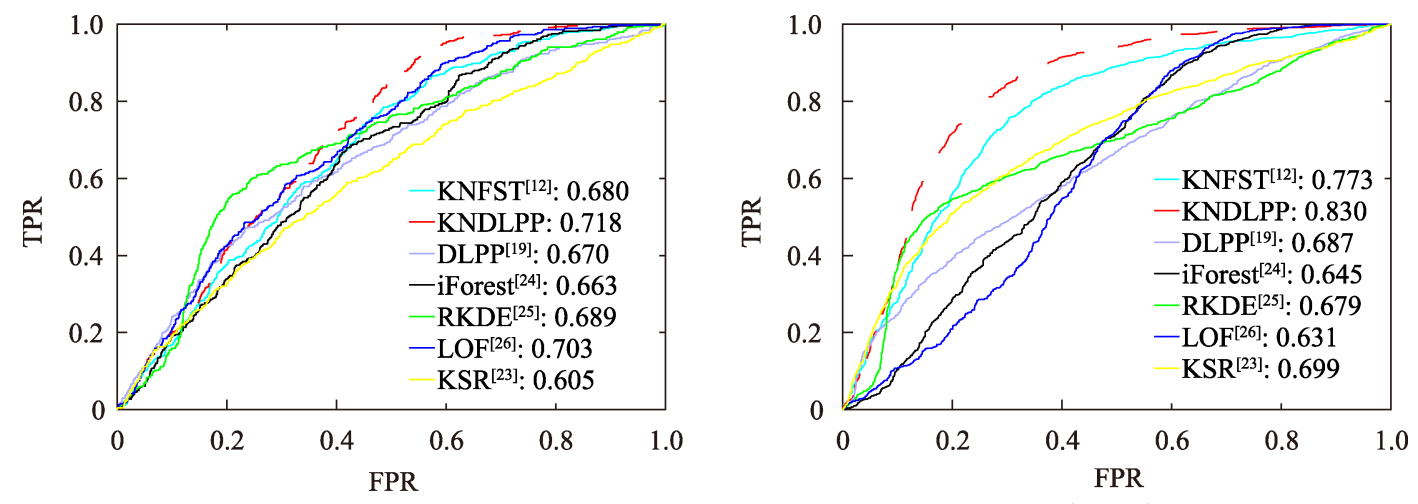

a. Glass

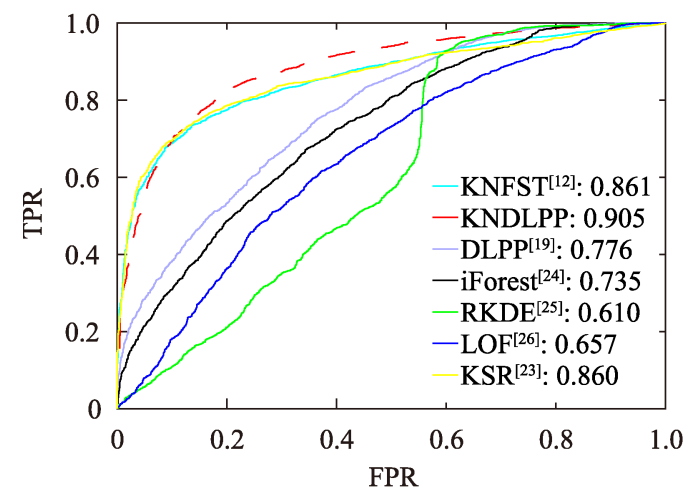

b. Seeds

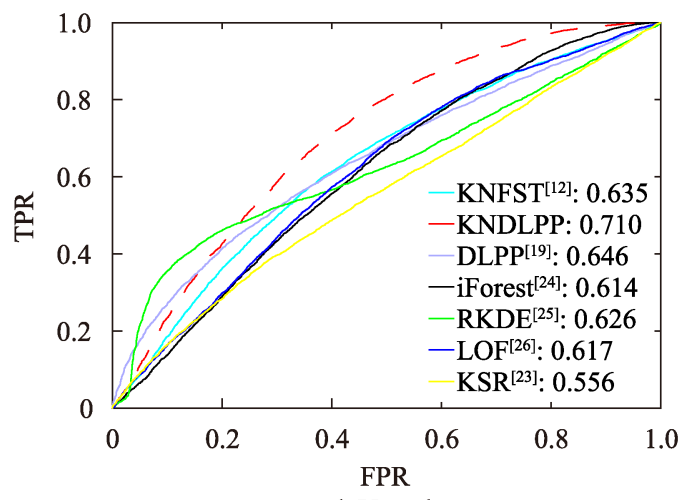

c. Ionosphere

d. Vowel

图 7 UCI 数据集 $30 \%$ 噪声程度下的 ROC 曲线及平均 AUC 值对比 

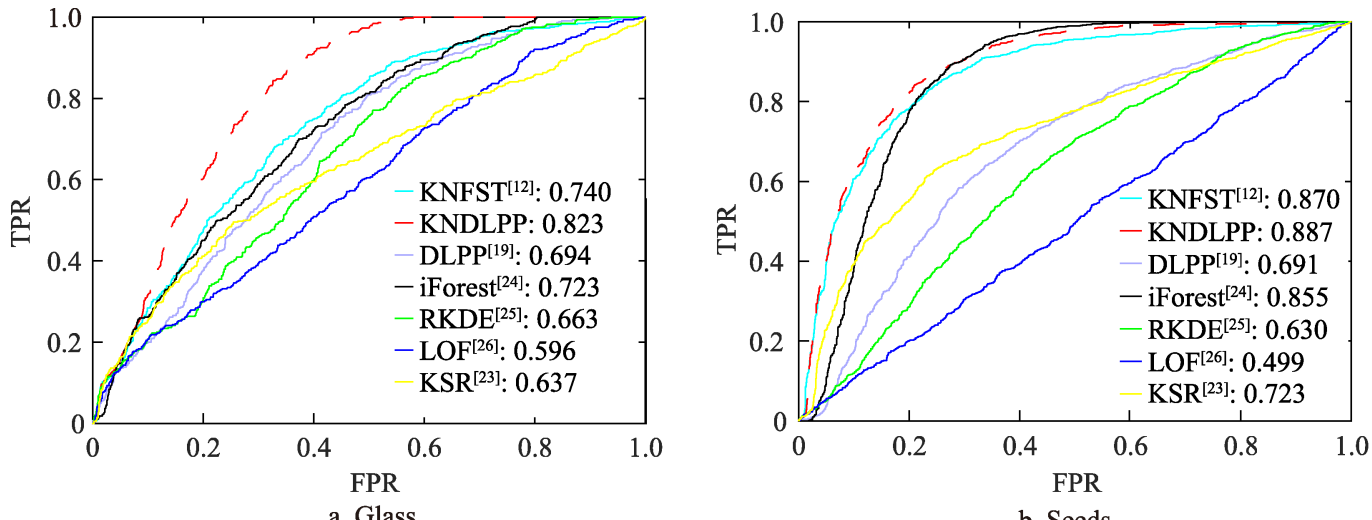

b. Seeds
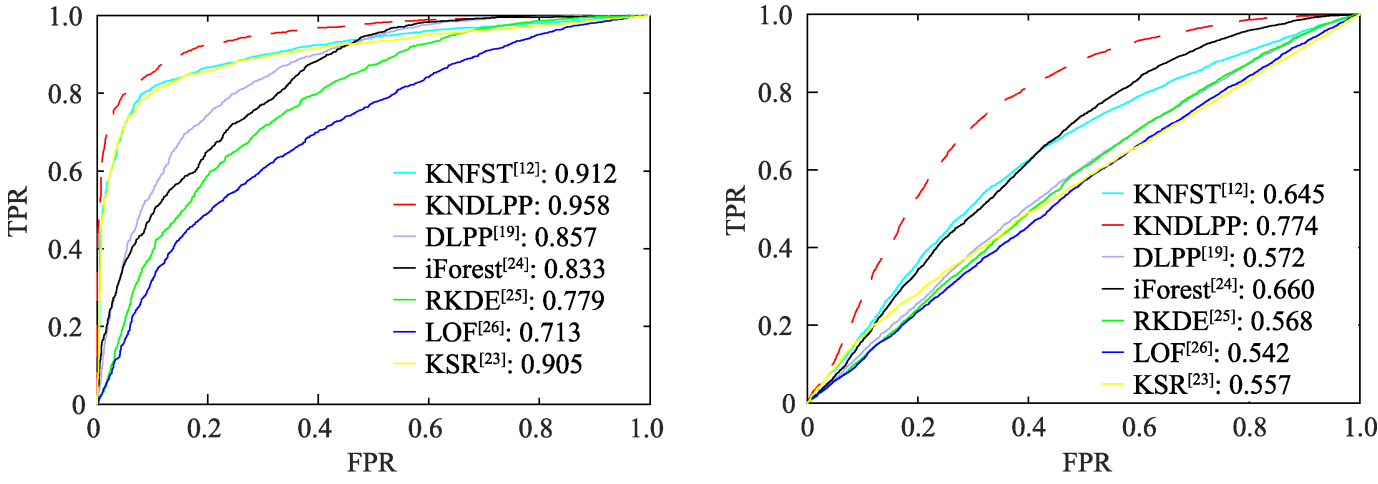

c. Ionosphere

d. Vowel

图 8 UCI 数据集扩展维度噪声下的 ROC 曲线及平均 AUC 值对比

观察图 5 图 8 中的 ROC 曲线和平均 AUC 值 可知, KNDLPP 均取得了整体最优的新类检测性 能. 与第 3.3 节的实验分析一致, 由于 iForest 和 LOF 均在数据原欧几里得空间对已知类分布进行 学习, RKDE 在其所映射的核空间对已知类分布进 行学习，三者均视原欧几里得空间或核空间的特 征维度同等重要,未充分挖掘数据的潜在结构信 息, 性能较差. 而受噪声影响, KSR 和 KNFST 这两 者在图 6 中的 Glass 和 Vowel 数据集上的性能甚至 低于 RKDE. 对比图 5 图 7 中同噪声类型下不同噪 声程度的结果, 随着噪声程度增加, 各算法性能均 有所下降. 这一现象证实了噪声样本对新类检测 的负面影响，也表明了 KNDLPP 研究思路的正确 性. 在图 8 中, 对维度扩展噪声, 由于 iForest 算法 的树结构具有特征选择功能, 所以其性能比 LOF 和 KSR 这两者更好. 综合以上分析, KNDLPP 可有 效地控制噪声样本的影响, 从数据的局部信息和 复杂结构角度提升了类别的可分性，在本次实验 中具有良好的新类检测性能.

\section{4 结 语}

本文提出了一种基于核零空间判别局部保持
投影的新类检测算法 KNDLPP. 该算法利用核函 数映射可刻画数据的复杂结构, 采用距离加权机 制可降低离群噪声样本的负面影响, 通过类内零 空间信息提升了类别之间的可分性，从 3 个角度提 升了新类检测的性能. 基于 7 个 UCI 数据集, 以及 Banana, Moon, Binalpha_uni 和 MSRA25_uni 数据 集的新类检测实验表明, KNDLPP 能根据已知类分 布学习一个判别性的变换, 在无噪和有噪条件下 均能较好刻画已知类和未知类之间的相似性, 具 有较好的有效性和鲁棒性. 相对特征噪声而言, 标 签噪声给样本分布带来的有偏性更为严重, 如何 在标签噪声情况下提升新类检测性能是未来可继 续探索的研究方向.

\section{参考文献(References):}

[1] Pimentel M A F, Clifton D A, Clifton L, et al. A review of novelty detection[J]. Signal Processing, 2014, 99: 215-249

[2] Perner P. Concepts for novelty detection and handling based on a case-based reasoning process scheme[J]. Engineering Applications of Artificial Intelligence, 2009, 22(1): 86-91

[3] Carino J A, Delgado-Prieto M, Zurita D, et al. Enhanced industrial machinery condition monitoring methodology based on novelty detection and multi-modal analysis[J]. IEEE Access, 2016, 4: 7594-7604 
[4] Ziaja A, Antoniadou I, Barszcz T, et al. Fault detection in rolling element bearings using wavelet-based variance analysis and novelty detection[J]. Journal of Vibration and Control, 2016, 22(2): 396-411

[5] Hayat M Z, Basiri J, Seyedhossein L, et al. Content-based concept drift detection for email spam filtering[C] // Proceedings of the 5th International Symposium on Telecommunications. Los Alamitos: IEEE Computer Society Press, 2010: 531-536

[6] Wang H X, Fan W, Yu P S, et al. Mining concept-drifting data streams using ensemble classifiers[C] //Proceedings of the 9th ACM SIGKDD International Conference on Knowledge Discovery and Data Mining. New York: ACM Press, 2003: 226-235

[7] Markou M, Singh S. Novelty detection: a review-part 1: statistical approaches[J]. Signal Processing, 2003, 83(12): 24812497

[8] Scott D W, Thompson J R. Probability density estimation in higher dimensions[C] //Proceedings of Computer Science and Statistics: Proceedings of the 15th Symposium on the Interface. Netherlands: Elsevier Science Publishers, 1983, 528: 173-179

[9] Zhu X F, Zhang S C, Hu R Y, et al. Local and global structure preservation for robust unsupervised spectral feature selection[J]. IEEE Transactions on Knowledge and Data Engineering, 2017, 30(3): 517-529

[10] Yang L, Jin R, Sukthankar R, et al. An efficient algorithm for local distance metric learning[C] //Proceedings of the 21st National Conference on Artificial Intelligence. Palo Alto: AAAI Press, 2006: 543-548

[11] Foley D H, Sammon J W. An optimal set of discriminant vectors[J]. IEEE Transactions on Computers, 1975, C-24(3): 281-289

[12] Bodesheim P, Freytag A, Rodner E, et al. Kernel null space methods for novelty detection[C] //Proceedings of the IEEE Conference on Computer Vision and Pattern Recognition. Los Alamitos: IEEE Computer Society Press, 2013: 3374-3381

[13] Tang J J, Tian Y J, Liu X H. LGND: a new method for multi-class novelty detection[J]. Neural Computing and Applications, 2019, 31(8): 3339-3355

[14] Guo Y F, Wu L D, Lu H, et al. Null Foley-Sammon transform[J]. Pattern Recognition, 2006, 39(11): 2248-2251
[15] Zhang L, Xiang T, Gong S G. Learning a discriminative null space for person re-identification[C] //Proceedings of the IEEE Conference on Computer Vision and Pattern Recognition. Los Alamitos: IEEE Computer Society Press, 2016: 1239-1248

[16] Huang X Y, Xu J L, Guo G. Incremental kernel null Foley-Sammon transform for person re-identification[C] // Proceedings of the 24th International Conference on Pattern Recognition. Los Alamitos: IEEE Computer Society Press, 2018: $1683-1688$

[17] Liao S C, Hu Y, Zhu X Y, et al. Person re-identification by local maximal occurrence representation and metric learning[C] // Proceedings of the IEEE Conference on Computer Vision and Pattern Recognition. Los Alamitos: IEEE Computer Society Press, 2015: 2197-2206

[18] Yang L P, Gong W G, Gu X H, et al. Null space discriminant locality preserving projections for face recognition[J]. Neurocomputing, 2008, 71(16-18): 3644-3649

[19] Yu W W, Teng X L, Liu C Q. Face recognition using discriminant locality preserving projections[J]. Image and Vision Computing, 2006, 24(3): 239-248

[20] He X F, Niyogi P. Locality preserving projections[C] // Proceedings of Advances in Neural Information Processing Systems. Cambridge: MIT Press, 2004: 153-160

[21] Dua D, Graff C. UCI machine learning repository[OL]. [2020-08-04]. http://archive.ics.uci.edu/ml

[22] Domingues R, Michiardi P, Barlet J, et al. A comparative evaluation of novelty detection algorithms for discrete sequences[OL]. [2020-08-04]. http://arxiv.org/pdf/1902.10940

[23] Cai D, He X F, Han J W. Speed up kernel discriminant analysis[J]. The VLDB Journal, 2011, 20(1): 21-33

[24] Liu F T, Ting K M, Zhou Z H. Isolation forest[C] //Proceedings of the 8th IEEE International Conference on Data Mining. Los Alamitos: IEEE Computer Society Press, 2008: 413-422

[25] Kim J S, Scott C D. Robust kernel density estimation[J]. Journal of Machine Learning Research, 2012, 13(82): 25292565

[26] Breunig M M, Kriegel H P, Ng R T, et al. LOF: identifying density-based local outliers[C] //Proceedings of the ACM SIGMOD International Conference on Management of Data. New York: ACM Press, 2000: 93-104 